\title{
Wake of a beam passing through a diffraction radiation target
}

\author{
Dao Xiang, ${ }^{*}$ Wen-Hui Huang, and Yu-Zheng Lin \\ Department of Engineering Physics, Tsinghua University, Beijing, China, 100084
}

Sung-Ju Park and In Soo Ko

Pohang Accelerator Laboratory, POSTECH, Pohang, Korea, 790784

(Received 28 October 2007; published 15 February 2008)

\begin{abstract}
Diffraction radiation (DR) is one of the most promising candidates for electron beam diagnostics for International Linear Collider and $\mathrm{x}$-ray free electron lasers due to its nonintercepting characteristic. One of the potential problems that may restrict its applications in real-time monitoring beam parameters is the wakefield generated by the presence of the DR target. In this paper, a comparative study of the wakefield and the backward DR (BDR) field is performed to clarify the relationship between them. The wakefield is studied with a particle-in-cell code MAGIC and the DR field is calculated based on virtual photon diffraction model. It is found that they have the same frequency spectrum and angular distribution, which indicates that the difference only exists in the subjective terminology. The longitudinal and transverse wake for a beam passing through a DR target is calculated for a general case when the beam's velocity is smaller than that of light. The resulted emittance growth and energy spread growth due to the short range wakefield is estimated and found to be permissible. In real measurement where BDR propagates in the direction perpendicular to the trajectory, it may add a transverse kick to the beam as a requirement of momentum conservation. The kick is found to be large enough to degrade the performance of accelerator driven facilities and needs to be corrected.
\end{abstract}

DOI: 10.1103/PhysRevSTAB.11.024001

PACS numbers: 41.60.- $\mathrm{m}, 41.75 . \mathrm{Ht}, 41.20 .-\mathrm{q}$

\section{INTRODUCTION}

There are growing interests in developing nonintercepting and real-time methods for beam diagnostics. The nonintercepting feature enables the method to measure high intensity beam where the heat deposition could destroy the target as is encountered by all the intercepting methods, e.g., optical transition radiation, wire scanner, phosphor screen, etc. The real-time capability is also highly preferable because it facilitates the machine optimization and allows monitoring beam parameters during operation. Diffraction radiation (DR) is considered as one of the most promising methods that may concurrently have both capabilities [1-5,5-8].

While its nonintercepting feature has been widely accepted, its real-time capability still needs investigation. Because of the wakefield generated by the presence of the DR target, there is concern on whether the wakefield would greatly deteriorate the beam qualities [9].

The DR is produced by the presence of optical inhomogeneity which induces changing currents that give rise to radiation. It may also be viewed as wakefield generated by a charged particle electromagnetically interacting with its surroundings [10]. Because of the fact that wakefield is generally studied near to the surroundings while the radiation field is typically treated in far field, it is hard to clarify the relationship between them. For some simple cases, this issue may be studied analytically $[11,12]$. For example, in our previous work [11], transition radiation (TR) has been

\footnotetext{
*xiangdao@tsinghua.org.cn
}

derived with the orthogonal mode expansion method that is widely used in wakefield calculations. A similar problem has been studied in [12] where the wake and impedance associated with the TR target is calculated for the ultrarelativistic case where the beam's velocity is assumed to be that of light. However, it appears difficult to extend their method to handle the wakefield of the DR target due to the complex boundary condition. In order to clarify the relationship between wakefield and TR or DR field, it is best to study the general case when the beam's velocity is smaller than that of light, because the properties of TR and DR depend on the beam's energy. Thus, one cannot compare the results from a conventional wakefield calculation with that of DR which is obtained under some specific energy.

In order to clarify the relationship between the DR field and the wakefield, we studied them in the near field for a general case when the beam's velocity is smaller than that of light. By setting sufficient monitors, the frequency spectrum and angular distribution of the wakefield are obtained from the particle-in-cell (PIC) code MAGIC [13] where the electromagnetic field is calculated from first principle with a finite difference approach in conjunction with the Lorentz motion of the electron beam. The results are compared with that of backward DR (BDR) obtained with a diffraction model that treats DR as superposition of virtual photons emitted from the target. When comparisons are made between them, good agreement is achieved, which indicates that the DR field is just the geometric wakefield associated with the target and the difference only exists in the subjective terminology. For practical cases, the target is tilted by 45 degrees with respect to 
the beam's trajectory and the main part of the BDR is extracted out from the acceleration tube through a window, thus its application in beam diagnostics would not affect the subsequent beam.

Nevertheless, one should note it is impossible to obtain information from the beam without perturbing it. In this paper it is found that the main perturbation caused by BDR is to add a transverse kick to the beam which is a requirement of momentum conservation. For typical parameters of International Linear Collider (ILC) and x-ray free electron lasers (XFEL), it is found that the kick could significantly degrade their performances and needs to be corrected. Furthermore, the forward DR (FDR) generated by the head of the bunch could catch up with the tail of the same bunch and interact with it. The perturbation caused by FDR is to cause a slight growth of the projected emittance and energy spread, which are found to be permissible.

It is worth pointing out that in real measurement the DR target is tilted by 45 degrees with respect to the beam's trajectory while for simplicity in our simulation and calculation the target is perpendicular to it. Our calculation for wakefield and impedance is justified because the FDR is independent of the inclined angle of the target and it is the FDR rather than BDR that catches up with the beam and affects the emittance and energy spread.

The remainder of this paper is organized as follows: In Sec. II we introduce the Fresnel-Kirchhoff diffraction model of virtual photons from which the DR field in near field is obtained. Section III describes the comparative study of DR field and wakefield with a powerful PIC code. After the equivalence of DR field and wakefield has been demonstrated, we then proceed in Sec. IV to consider the wake and impedance of a bunch passing through a DR target. The perturbations to electron beam's trajectory, energy spread, and projected emittance during the use of DR for beam diagnosis are investigated in Sec. V. The conclusions and discussions are summarized in Sec. VI.

\section{FRESNEL-KIRCHHOFF DIFFRACTION MODEL OF VIRTUAL PHOTONS}

When the target is infinitely stretched and the observation is made in the far field, the DR problem could be handled by solving Maxwell's equations [14]. But for practical conditions neither the target for generating TR and DR is infinitely large nor the observation could always be made in the far field, the properties of DR sometimes could largely deviate from that studied in [14].

The Fresnel-Kirchhoff diffraction model could be used to effectively treat this problem [15-17]. In this model, the field of an electron is quantized into virtual photon which is the Fourier transform of its time-dependent field. The virtual photon is locked to the electron and cannot propagate freely. When transmitted through or reflected by the metallic target, the virtual photon can be set free from the electron and convert to real photons propagating along the direction of velocity (FDR) and the specular reflection direction (BDR).

In SI units and cylindrical coordinates where the $z$-axis coincides with the electron's trajectory, the field generated by a relativistic electron is well known,

$$
E_{r}(z, r, t)=\frac{e \gamma}{4 \pi \varepsilon_{0}} \frac{r}{\left[r^{2}+\gamma^{2}(z-c t)^{2}\right]^{3 / 2}},
$$

where $r$ is the minimum distance from the observation point to the trajectory of the electron, $\gamma$ is the Lorentz factor, $z-c t$ is the projected distance from the electron to the observation point in the velocity direction. Consider a circular aperture in a perfectly conducting metal plate which is located in the $z=0$ plane, and we assume the electron passes through the aperture at the time $t=0$. The transverse electric field of the virtual photon with angular frequency $\omega$ is found by Fourier transform,

$$
E_{r}(r, \omega)=\int_{-\infty}^{\infty} E_{r}(0, r, t) e^{i \omega t} d t=\frac{e}{\varepsilon_{0} c \gamma \lambda} K_{1}\left(\frac{2 \pi r}{\gamma \lambda}\right),
$$

where $K_{1}(x)$ is the first order modified Bessel function of the second kind. For most of the cases of interest, we have $\gamma \gg 1$ and only the transverse field is important. The effective size of the virtual photon disk is found to be about $\sigma=(4 / \pi) \gamma \lambda$ [17]. So given an aperture, the virtual photon that would suffer strong reflection or diffraction and converts to real photons should be those for which the effective size is larger than the aperture.

Let us assume the radius of the aperture is $a$ and the outer radius of the conducting plate is $b$. We can define three regions in the plate plane: S1 denotes the aperture, S2 denotes the opaque plate, and $\mathrm{S} 3$ denotes the outer regions of the plane, as shown in Fig. 1.

When an electron passes through the aperture, the field at the points of the aperture and the outer region is assumed to be the same as what would be when the conducting plate is absent. Take the points of the aperture and the outer

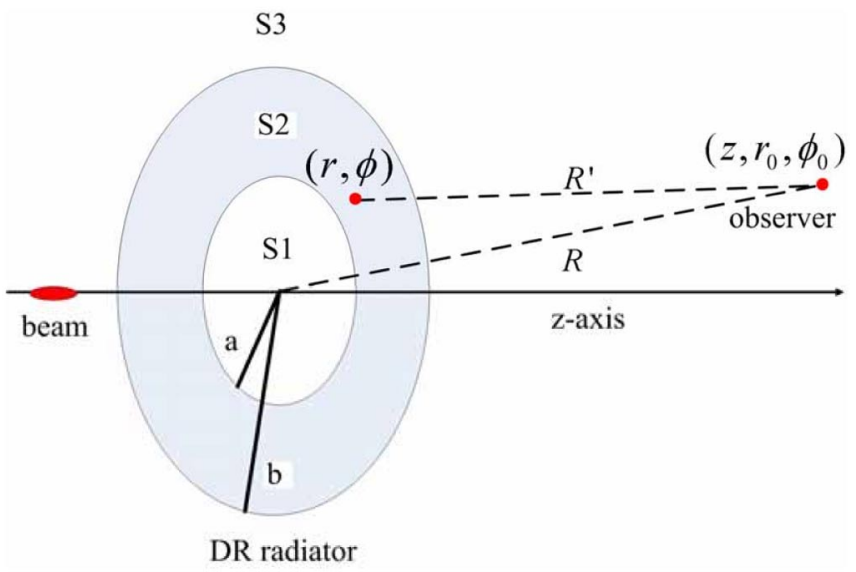

FIG. 1. (Color) Schematic show of the diffraction model. 
region as secondary waves, the field for a given frequency component in the space $z>0$ could be found by FresnelKirchhoff integration [18],

$$
\tilde{E}_{r}(z, r, \omega)=-\frac{i}{2 \lambda} \iint_{S 1+S 3} E_{r}(r, \omega)(1+\cos \chi) \frac{e^{i k R^{\prime}}}{R^{\prime}} d S,
$$

where $R^{\prime}$ is the distance from some point of the secondary waves to the observation point and $\chi$ is the angle between $R^{\prime}$ and the aperture central line. The forward DR field is found by subtracting the particle field [Eq. (3), taking the limit $S 1 \rightarrow \infty$ ] and can be written as

$$
\tilde{E}_{r}(z, r, \omega)_{\mathrm{DR}}=\frac{i}{2 \lambda} \iint_{S 2} E_{r}(r, \omega)(1+\cos \chi) \frac{e^{i k R^{\prime}}}{R^{\prime}} d S .
$$

As for backward DR, it should be considered as the reflected virtual photon from S2. The boundary condition requires the field on $\mathrm{S} 2$ to equal the particle field in magnitude and opposite in sign. The backward DR is found after performing the Fresnel-Kirchhoff integration on S2 and is also described by Eq. (4).

The distance from some point of the secondary wave $(0, r, \phi)$ to the observation point $\left(\mathrm{z}, r_{0}, \phi_{0}\right)$ can be approximately written as

$$
R^{\prime} \approx R-\sin \theta r \cos \left(\phi-\phi_{0}\right)+r^{2} / 2 R,
$$

where $R=\sqrt{z^{2}+r_{0}^{2}}$ is the distance from the observation point to the center of the aperture, $\sin \theta=r_{0} / R$. When an electron center passes through the aperture, substituting Eq. (5) into Eq. (4) and under the paraxial assumption, we have

$$
\begin{aligned}
\tilde{E}_{r}(z, r, \omega)_{\mathrm{DR}} \approx & \frac{2 \pi e e^{i k R}}{\varepsilon_{0} c \gamma \lambda^{2} R} \\
& \times \int_{a}^{b} r K_{1}(k r / \gamma) J_{1}(k \sin \theta r) e^{i k r^{2} / 2 R} d r .
\end{aligned}
$$

It is well known that, when $R \gg r_{\max }^{2} / \lambda$, we can drop the term $\exp \left(i k r^{2} / 2 R\right)$ in Eq. (6) while still maintaining the accuracy of the calculation [18]. In this case we can say the observation is in the far field. Note the fact that the virtual photon disk has an effective radius of about $\gamma$, so the far field condition is satisfied when $R \gg \gamma^{2} \lambda / 4 \pi^{2}$.

Let us first consider a simple case where the observation is made in the far field. After some mathematical manipulation, Eq. (6) is found to be

$$
\tilde{E}_{r}(z, r, \omega)_{\mathrm{DR}}=\frac{e e^{i k R}}{2 \pi \varepsilon_{0} c \gamma R} \frac{1}{\sin ^{2} \theta+\gamma^{-2}}[\mathrm{Y}(b)-\Upsilon(a)]
$$

and

$$
\begin{aligned}
\Upsilon(x)= & k \sin \theta x J_{2}(k \sin \theta x) K_{1}(k x / \gamma) \\
& -(k x / \gamma) J_{1}(k \sin \theta x) K_{2}(k x / \gamma) .
\end{aligned}
$$

Equation (7) indicates that the DR extends from very low frequency up to $\gamma c / a$ beyond which the intensity decreases dramatically. This can be explained with Eq. (1) which shows that the time for the target edge to feel a considerable field is $a / \gamma c$. This is also the DR emission time in which the induced currents are generated in response to the transient Coulomb field of the relativistic electron. The higher the beam's energy, the shorter the emission time and the higher the frequency of DR can extend.

The energy per angular frequency per solid angle for DR is found to be

$$
\begin{aligned}
\frac{d^{2} W}{d \omega d \Omega} & =\frac{\varepsilon_{0} c}{\pi}\left|R \tilde{E}_{r}\right|^{2} \\
& =\frac{e^{2}}{4 \pi^{3} \varepsilon_{0} c \gamma^{2}} \frac{1}{\left(\sin ^{2} \theta+\gamma^{-2}\right)^{2}}[\Upsilon(b)-\Upsilon(a)]^{2} .
\end{aligned}
$$

Some comments on the application range of Eq. (6) are necessary. First, the reflection or diffraction of virtual photons is related to the surface currents. This concept only holds when radiation frequency is much smaller than plasma frequency of the conducting plate. So the diffraction model can only be applied to handle the frequency component ranging from microwave to optical rather than UV or x-ray. Second, Eq. (6) is valid if the observation is made at a distance long compared to the radiation wavelength. Third, the assumption that the field in the aperture is the same as what would be in the absence of the plate is a suitable approximation when radiation wavelength is smaller than the aperture radius [19]. If the wavelength is much longer than the aperture size, Eq. (6) may become inaccurate.

In using Eq. (2) we have assumed the electron flies from $-\infty$, so the phase only depends on $z$. However, for our simulation case as we will show in the next section, the electron has a starting point. Considering that the wave front is a spherical surface, the phase of the virtual photon also depends on $r$. A modified diffraction model for the electron that starts at finite distance is necessary. The phase term of the virtual photon field is found to be $\exp (i k z+$ $i k r^{2} / 2 z$ ). It is worth pointing out that it takes some time for the virtual photon to develop the plane wave properties. The time depends on beam energy and frequency of the virtual photon of interest. For the component whose wavelength is $\lambda$, the maximum $r$ of importance is in the order of $\gamma \lambda$, so the time can be quantified with the condition $k \gamma^{2} \lambda^{2} / 2 c t_{0} \ll 1$. The corresponding distance needed for the virtual photon to develop the plane wave property can be written as

$$
z_{0}=c t_{0} \gg \gamma^{2} \lambda .
$$


It is interesting to note that this is also the distance beyond which the far field assumption holds. We will show in the section below that only when the additional phase term is taken into account can the diffraction model give an accurate description for the DR field from an electron bunch that starts at finite distance.

\section{SIMULATION MODEL SETUP AND RESULTS}

The geometry for the simulation is shown in Fig. 2. We have used cylindrical coordinate systems for our 2D simulation, thus any parameter is symmetric in the azimuthal direction. The radiator is a perfectly conducting circular disk located at $z=0$ with inner radius $a=4 \mathrm{~mm}$ and outer radius $b=200 \mathrm{~mm}$. The cathode is located at $z=$ $-199 \mathrm{~mm}$ from which a perfectly parallel electron beam is emitted with an energy of $10 \mathrm{MeV}$. The beam distribution is Gaussian in the longitudinal direction with rms bunch length $\sigma_{z}=3 \mathrm{ps}$ and uniform in the transverse direction. Its radius is chosen to be $1 \mathrm{~mm}$ which is much smaller than that of the aperture in order to make the assumption that all the electrons center pass through the aperture valid and facilitate the comparisons with the results predicted by the virtual photon diffraction model. The peak current of the beam is $3 \mathrm{~A}$ and the Gaussian distribution is truncated at $\pm 3 \sigma_{z}\left(1.41 \times 10^{8}\right.$ electrons per bunch and 20000 macroparticles are used in the simulation). The boundaries of the simulation setup are free space regions to minimize electromagnetic reflection. The grids are very delicate in the region where the currents are present (step size of $0.25 \mathrm{~mm}$ and $0.1 \mathrm{~mm}$ in longitudinal and transverse directions), a bit larger in other regions (step size of $0.25 \mathrm{~mm}$ in both directions). The time step is chosen to satisfy the Courant condition and is $207 \mathrm{fs}$.

The 31 monitors in the left of the radiator with an angular interval of $0.02 \mathrm{rad}$ in the range [0.01, 0.61] rad are used to record the time signals at the specific positions. The monitors are located on the spherical surface with radius $100 \mathrm{~mm}$ as shown in Fig. 2. As an example, the whole time signal recorded by the monitor at $\theta=0.09 \mathrm{rad}$ is shown in Fig. 3.

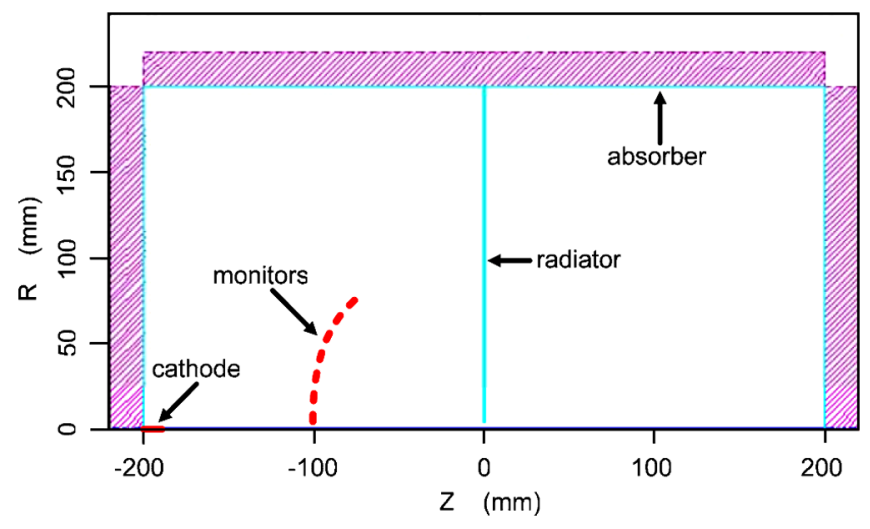

FIG. 2. (Color) Geometry of the simulation.

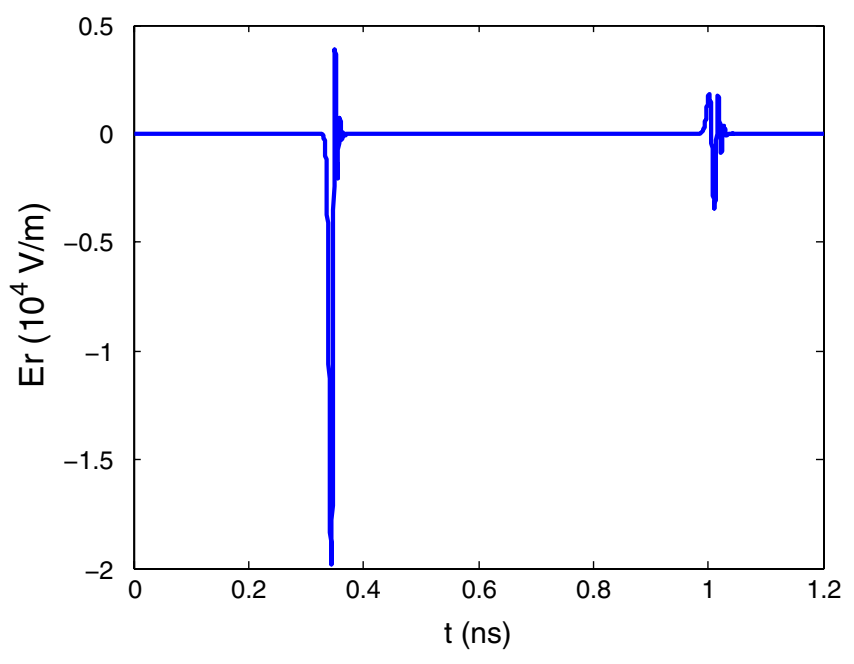

FIG. 3. (Color) Time signal recorded by one monitor.

From Fig. 3 we clearly see that the monitor detected two separate signals. The first signal that starts from about 0.3 to $0.35 \mathrm{~ns}$ represents the beam's Coulomb field detected when the beam passes beneath the monitor and the second signal that starts from $0.95 \mathrm{~ns}$ to approximately $1.05 \mathrm{~ns}$ should represent the wakefield generated by the presence of the target. The delay of the second signal is dominated by causality that the field needs about $0.7 \mathrm{~ns}$ to reach the target and another $0.3 \mathrm{~ns}$ to again reach the monitors.

Since we are only interested in the wakefield, we will only consider the second signal. The power spectrum of the second signal recorded by the monitor at $\theta=0.09 \mathrm{rad}$ is shown in Fig. 4.

For convenience of comparison, the frequency spectrum of the wakefield obtained from the Fresnel-Kirchhoff diffraction model and the modified Fresnel-Kirchhoff diffrac-

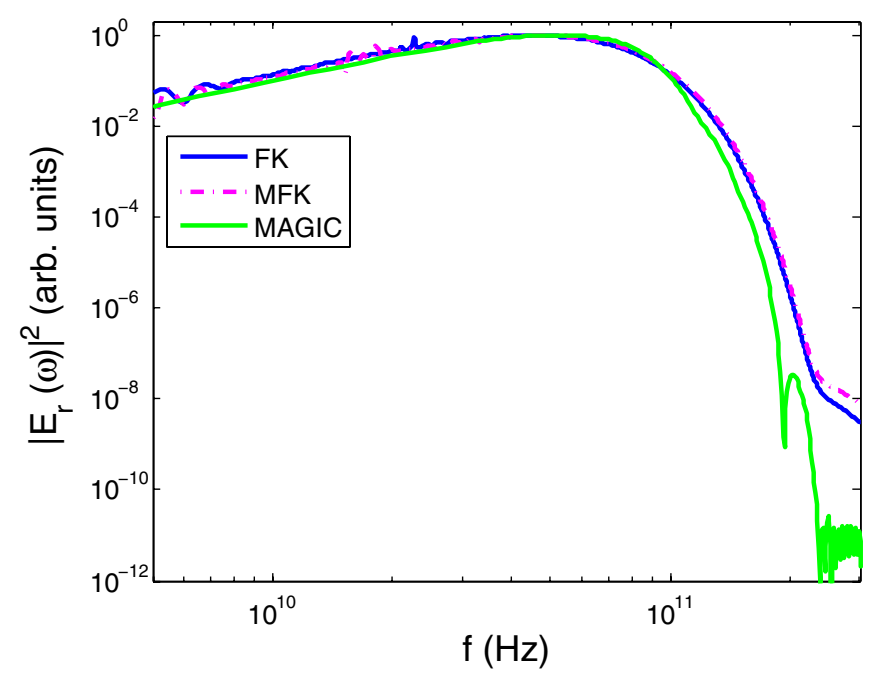

FIG. 4. (Color) Wakefield power spectrum from FresnelKirchhoff diffraction model (FK), from modified FresnelKirchhoff diffraction model (MFK) and MAGIC simulation. 
tion model are also shown in Fig. 4. The agreement is quite good for the low frequency component where MAGIC can provide an accurate calculation when the wavelength is much larger than the grid size. However, as for high frequency for which the wavelength is comparable or smaller than grid size, a significant difference occurs which is due to the fact that MAGIC cannot provide valid data for these components. From Fig. 4 we conclude that, within the frequency range where MAGIC can provide valid data, the wakefield and the DR field have the same spectrum (or equivalently the same time structure) for some specific observation point.

When combining the spectrum intensity with the monitors, we are allowed to obtain the wakefield spectral angular distribution for various frequency components. The signals from the 31 monitors are Fourier transformed first,

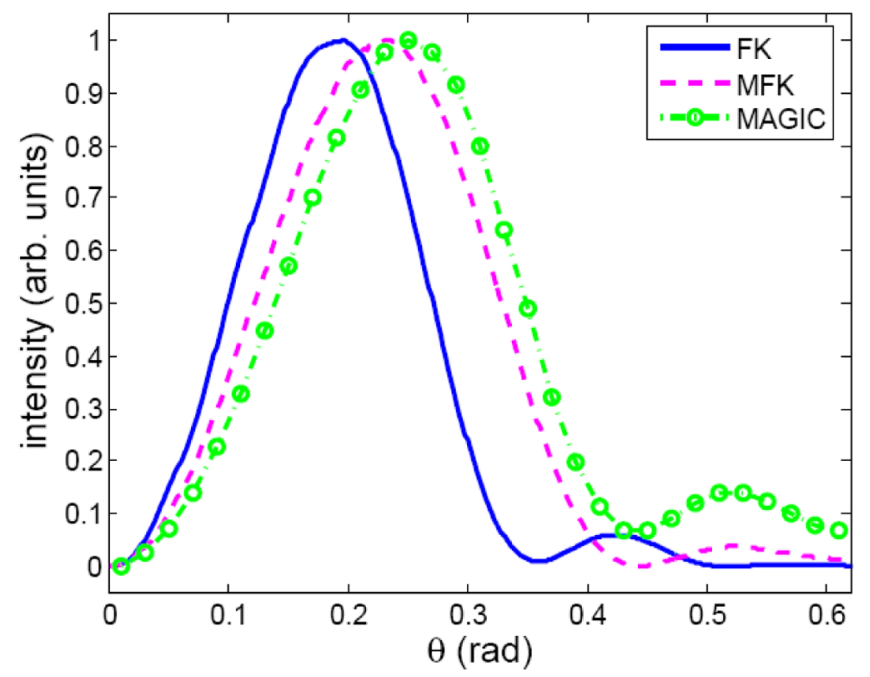

(a)

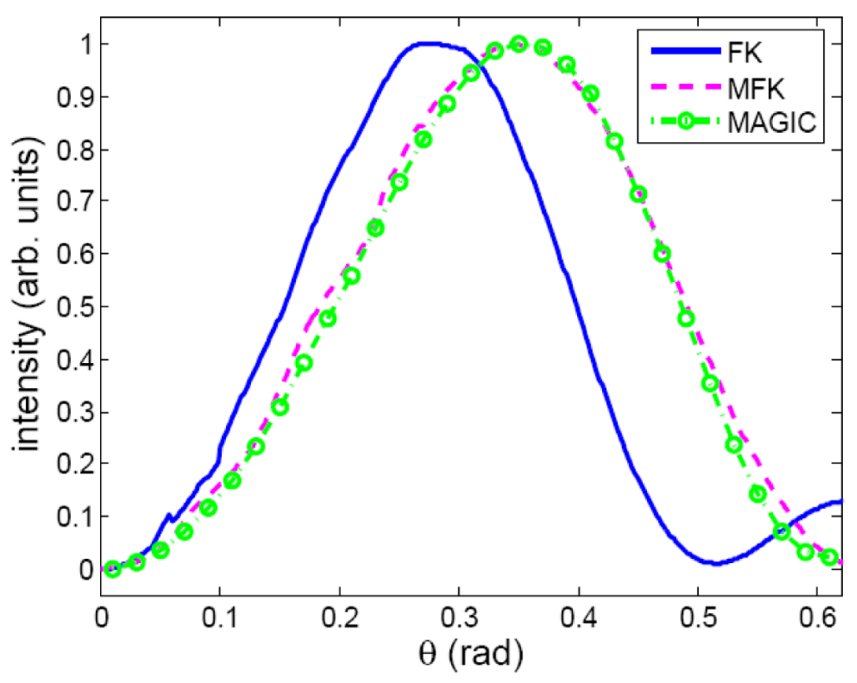

(b)

FIG. 5. (Color) Angular distribution of the wakefield and DR for various frequency components. (a) $\lambda=6 \mathrm{~mm}$; (b) $\lambda=12 \mathrm{~mm}$. and the spectral intensity for $\lambda=6 \mathrm{~mm}$ and $\lambda=12 \mathrm{~mm}$ is extracted from each specific monitor to give the angular distribution for the wakefield. The results and that of the DR given by the diffraction model with the finite target size effect and near field effect taken into account are shown in Fig. 5.

As can be seen in Fig. 5, the spectral angular distribution of wakefield calculated by MAGIC is in good agreement with that of the DR predicted with diffraction model. Especially when the modified Fresnel-Kirchhoff model is used to account for the phase of the virtual photons, the agreement is excellent for the $\lambda=12 \mathrm{~mm}$ case while some tiny difference exists for $\lambda=6 \mathrm{~mm}$. It is worth pointing out that, for the component whose wavelength is much larger than the aperture size, say $\lambda=60 \mathrm{~mm}$, we found that the angular distribution from the diffraction model and that given by MAGIC differ again. This is due to the inability of the Fresnel-Kirchhoff diffraction model in describing the component whose wavelength is orders larger than the aperture size [19], for which the assumption that the field in the aperture is the same as what would be in the absence of the aperture does not hold.

From what have been studied above, we see that to a satisfactory extent the geometric wakefield associated with the DR radiator and the DR field have the same spectrum and obey the same angular distribution. Since the spectrum and angular distribution are sufficient in describing the field in 4D space, we conclude that the two concepts are unified and it seems they only differ in subjective terminology.

\section{WAKE AND IMPEDANCE OF DIFFRACTION RADIATION}

Let us first consider a simple case where a single electron center passes through a circular DR target with inner radius $a$ and outer radius $b$. The energy loss of a single electron during the passage of the aperture is related to the real part of the longitudinal coupling impedance as [20]

$$
\Delta E=\frac{e^{2}}{\pi} \int_{0}^{\infty} \operatorname{Re} Z_{\|}(\omega) d \omega,
$$

where $Z_{\|}(\omega)$ is defined as [21]

$$
Z_{\|}(\omega)=-\frac{1}{q} \int_{-\infty}^{\infty} E_{z}(z) e^{-i \omega z / v} d z
$$

Direct calculation for $Z_{\|}(\omega)$ from Eq. (11) is not trivial. Alternatively, we can relate the longitudinal impedance to the energy loss of the electron. Since the equivalence of wakefield and DR field has been demonstrated in Sec. III, we are allowed to calculate the energy loss by performing the integration of the DR energy per angular frequency in the whole space (including both forward DR and backward DR), so we have 


$$
\operatorname{Re} Z_{\|}(\omega)=\frac{2 \pi}{e^{2}} \int_{0}^{\pi / 2} \sin \theta d \theta \int_{0}^{2 \pi} d \phi \frac{d^{2} W}{d \omega d \Omega_{\mathrm{DR}}}
$$

Calculation for longitudinal impedance from Eq. (12) is in principle possible but may be cumbersome [22]. A more important thing is that during calculation of the angular distribution for DR we have used the paraxial assumption, so Eq. (8) is only accurate when the observation angle is much smaller than unity. However, performing integration in the whole space involves large observation angles, which if done with Eq. (8) may make the result inaccurate, especially for the moderately relativistic case. In addition, if one is also interested in the transverse impedance for which the calculation from Eq. (12) requires that the angular distribution of DR when an electron passes through the aperture with nonzero offset to the aperture center to be known. To find the DR angular distribution for a nonzero offset electron with the finite size of the target taken into account also seems to be a tough task.

Recalling the equivalence of the wakefield and DR field, a more tricky method in calculating the longitudinal impedance may be to directly calculate the energy contained by the virtual photons that are reflected by the DR target. We consider a general case when an electron passes through the aperture with an offset $\rho$ with respect to the aperture center, the longitudinal coupling impedance may be calculated as

$$
\begin{aligned}
\operatorname{Re}_{\|}(\omega)= & \frac{2 \pi}{e^{2}} \int_{0}^{2 \pi} d \varphi \int_{a}^{b} \frac{\varepsilon_{0} c}{\pi}\left|E_{r}(r, \omega)\right|^{2} r d r \\
= & \frac{\kappa^{2}}{2 \pi^{2} \varepsilon_{0} c} \int_{0}^{2 \pi} d \varphi \\
& \times \int_{a}^{b} K_{1}\left(\kappa \sqrt{r^{2}+\rho^{2}-2 r \rho \cos \varphi}\right)^{2} r d r,
\end{aligned}
$$

where $\kappa=k / \gamma$. It is safe to assume $\rho \ll a$, because during beam diagnosis, we do not expect the electron to hit the target. Note that [23]

$$
\begin{aligned}
\frac{K_{1}\left(\kappa \sqrt{r^{2}+\rho^{2}-2 r \rho \cos \varphi}\right)}{\sqrt{r^{2}+\rho^{2}-2 r \rho \cos \varphi}}= & \frac{2}{\kappa} \sum_{n=1}^{\infty} n \frac{I_{n}(\kappa \rho)}{\rho} \frac{K_{n}(\kappa r)}{r} \\
& \times \frac{\sin n \varphi}{\sin \varphi}
\end{aligned}
$$

If we further assume that $\kappa a \ll 1$, the first term dominates the infinite series in Eq. (14) and we may approximately write

$$
K_{1}\left(\kappa \sqrt{r^{2}+\rho^{2}-2 r \rho \cos \varphi}\right) \approx(1-\rho \cos \varphi / r) K_{1}(\kappa r)
$$

Substituting Eq. (15) into Eq. (13), we have

$$
\operatorname{Re} Z_{\|}(\omega)=\frac{\kappa^{2}}{2 \pi^{2} \varepsilon_{0} c} \int_{a}^{b} K_{1}(\kappa r)^{2}\left(2 \pi r+\pi \rho^{2} / r\right) d r
$$

The first term of Eq. (16) independent of the transverse position is the monopole longitudinal coupling impedance and is found to be [24]

$$
\begin{aligned}
Z_{\|}{ }^{0}(\omega)= & \frac{\kappa^{2}}{2 \pi^{2} \varepsilon_{0} c} \int_{a}^{b} K_{1}(\kappa r)^{2} 2 \pi r d r \\
= & \frac{\kappa^{2}}{2 \pi \varepsilon_{0} c}\left[a^{2} K_{0}(\kappa a) K_{2}(\kappa a)-b^{2} K_{0}(\kappa b) K_{2}(\kappa b)\right. \\
& \left.+b^{2} K_{1}(\kappa b)^{2}-a^{2} K_{1}(\kappa a)^{2}\right]
\end{aligned}
$$

Equation (17) is in good agreement with that obtained in [25] except for the different use of Bessel functions, but our calculation is much simpler. In Ref. [26], the impedance for a circular aperture which has infinite size is also studied. The result is similar to Eq. (17) except for the terms involving the outer radius of the aperture. We should note that the calculation for impedance with the outer radius of the aperture taken into account is necessary. It can be easily shown that Eq. (17) is well behaved when $\kappa$ tends to zero while that in [26] diverges. The monopole longitudinal impedance for a finite size circular aperture and infinite size circular aperture is calculated and shown in Fig. 6.

As can be seen in Fig. 6, the impedance is dependent on target size and beam energy. For the extreme case when the beam's velocity equals that of light, Eq. (17) can be reduced to

$$
\operatorname{Re} Z_{\|}^{0}(\omega)=\frac{Z_{0}}{\pi} \ln \frac{b}{a}
$$

where $Z_{0}=\sqrt{\mu_{0} / \varepsilon_{0}}$ is the characteristic impedance of

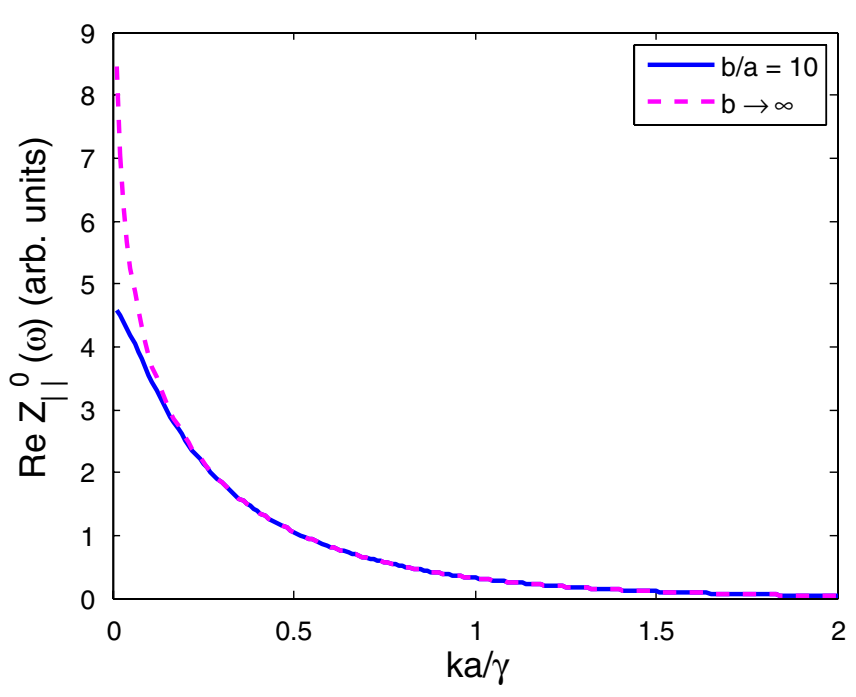

FIG. 6. (Color) Monopole longitudinal impedance for a circular DR target. 
free space. Equation (18) is just the well-known longitudinal impedance for a perfectly conducting circular thin collimator in high frequency obtained with the assumption $v=c[25,27]$. It is worth commenting on the application range of Eqs. (17) and (18). It is easy to see that the energy loss diverges if Eq. (18) holds when $\omega \rightarrow \infty$. We should first note that a uniformly moving electron will not emit radiation unless there are inhomogeneities. When the frequency of interest is much larger than the plasma frequency of the DR target, the permittivity of the DR target tends to unity and there is no big difference between the target and vacuum. So the field of very high frequency will not "see" the target. The plasma frequency is the upper limit for the application range of Eq. (18). Recall the fact that only those virtual photons whose effective size is larger than the aperture radius are converted to real photons and contribute to impedance, we can conclude that the upper limit for Eqs. (17) and (18) to be valid is $\min \left[\gamma c / a, f_{\text {plasma }}\right]$. The lower limit for Eqs. (17) and (18) to be valid is that the radiation wavelength is smaller than the aperture radius. When the wavelength is much larger than the aperture radius, the assumption that the field in the aperture is the same as what would be in the absence of the plate is no longer a suitable approximation and the FresnelKirchhoff diffraction model may give inaccurate results. So in Fig. 6 only the data for which $k a / \gamma \gg 1 / \gamma$ is meaningful.

The longitudinal wake can be calculated as

$$
W_{\|}(s)=\frac{2}{\pi} \int_{0}^{\infty} d \omega \operatorname{Re} Z_{\|}{ }^{0}(\omega) \cos \frac{\omega s}{c} .
$$

Here the convention is that $W_{\|}(s)$ is positive for energy loss. The longitudinal wake for a circular aperture with various outer radius is shown in Fig. 7,

The wake is also dependent on beam energy and target size, which indicates the necessity of a general study when the beam's velocity is a bit smaller than that of light. As can

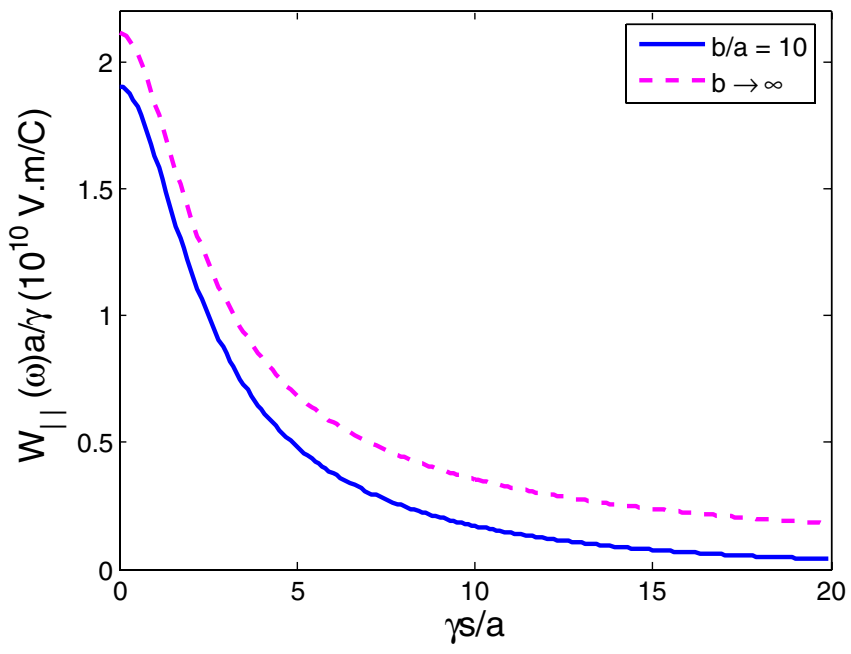

FIG. 7. (Color) Longitudinal wake for a circular aperture. be seen in Fig. 7, no electron behind gets accelerated. This wake is similar to that generated by a suddenly accelerated electron bunch [28]. When a single electron center passes through the circular aperture, it would lose energy due to generation of wakefield. The energy loss is related to the longitudinal wake as

$$
\Delta E=-\frac{1}{2} e W_{\|}\left(0^{-}\right)
$$

The term $1 / 2$ is due to the fundamental theorem of beam loading [29]. For the parameters used in the MAGIC simulation, Eq. (20) gives $0.8 \times 10^{-5} \mathrm{eV}$ for the energy loss when a single electron center passes through the circular aperture.

For a bunch with $N$ electrons, in addition to generation of DR, the electron loses energy due to interaction with the wakefield generated by all the electrons in front of it. The energy loss for some electron within the bunch can be calculated as

$$
\Delta E(s)=-W_{b \|}(s)=-N e^{2} \int_{s}^{\infty} d s^{\prime} f\left(s^{\prime}\right) W_{\|}\left(s^{\prime}-s\right),
$$

where $f(s)$ is the normalized longitudinal distribution of the bunch and $W_{b \|}(s)$ is referred as the longitudinal bunch wake. For the bunch used in the MAGIC simulation, the energy loss for electrons at various positions is calculated and shown in Fig. 8.

Since MAGIC performs a self-consistent calculation for the interaction between the electron beam and the scattered electromagnetic field, it is capable of monitoring the energy change of the electrons with various positions. We have recorded the energy evolution for each electron. Because of the fact that the beam is just moderately relativistic, the space charge is still able to cause an energy change for electrons with various positions within the

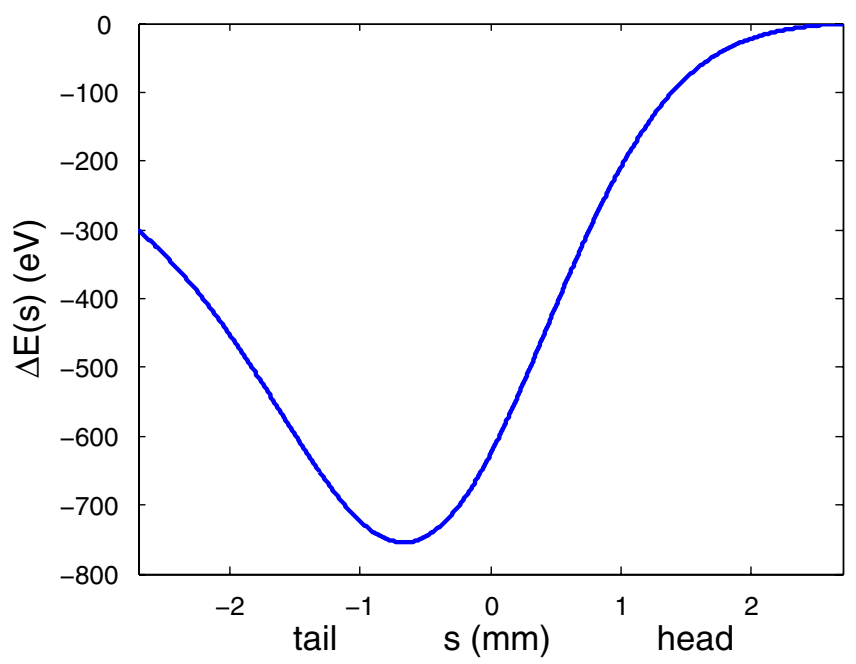

FIG. 8. (Color) Energy loss for electrons with various longitudinal positions in a bunch. 
bunch. To exclude the contribution from the space charge force, we performed another simulation in which there is no DR radiator. The longitudinal DR wake for the bunch is given by the difference of the two simulation results. The energy change for the electrons with various longitudinal positions recorded at various times is shown in Fig. 9.

From Fig. 9 we could see that before the electron beam arrives at the radiator (blue line), the two simulations give the same results for the energy evolution. After the bunch passes through the radiator, the wake from the head of the bunch catches up with the electrons in the tail of the bunch and starts to act. As the interaction continues, the energy loss gradually approaches $1 / 2$ of the longitudinal steadystate wake as described in Eq. (21) and Fig. 8. The term 1/2 arises from the fact that the simulation only considers the interaction between FDR and the electron beam. When BDR is generated, Maxwell's equations will not subtract the energy from the electron.

The total energy loss for a bunch can be calculated as

$$
\Delta E_{\mathrm{total}}=\frac{Q^{2}}{\pi} \int_{0}^{\infty} d \omega \operatorname{Re} Z_{\|}^{0}(\omega) F(\omega)
$$

With the parameters used in the MAGIC simulation, Eq. (22) gives an energy loss of about 12.5 nJ. From the MAGIC simulation, as can be seen in Fig. 9, the average energy loss for each electron is about $280 \mathrm{eV}$ and the corresponding total energy loss for the bunch is about $6.35 \mathrm{~nJ}$. If we take the energy of the BDR to be the same as that of FDR, the MAGIC simulation predicts an energy loss of about $12.7 \mathrm{~nJ}$ which is in good agreement with that obtained from Eq. (22).

From Eq. (16), the dipole longitudinal coupling impedance is given by

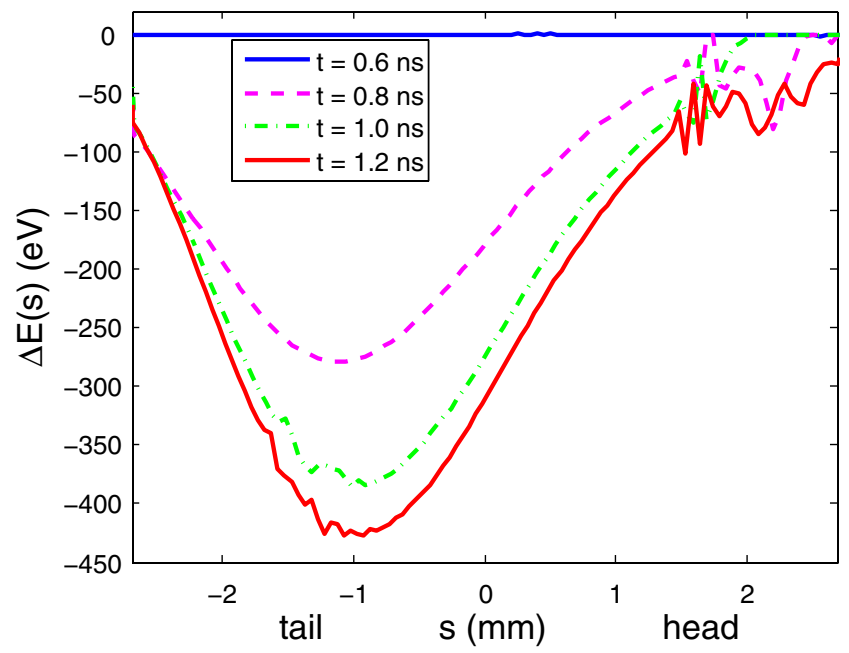

FIG. 9. (Color) Energy loss for electrons with various longitudinal positions in a bunch at various time.

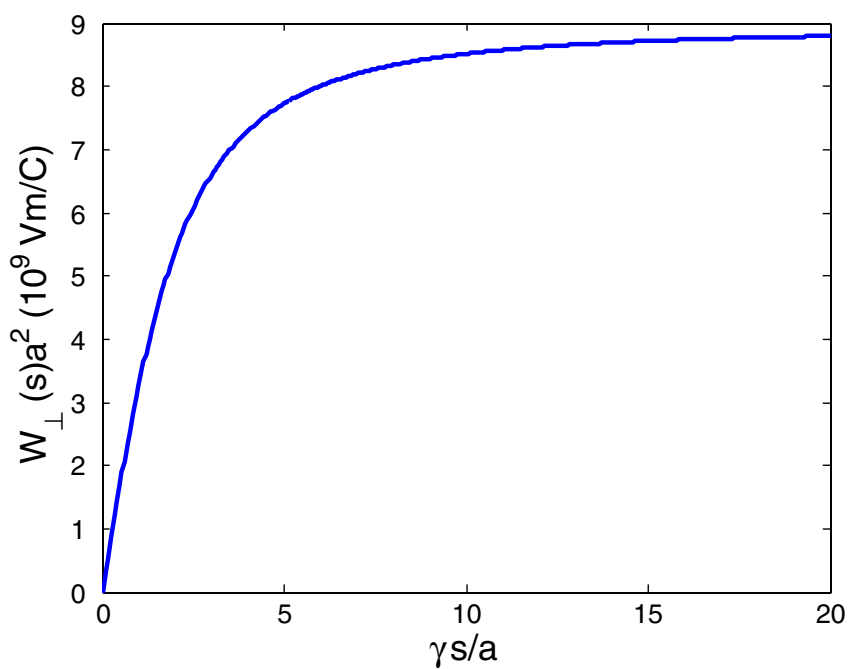

FIG. 10. (Color) Transverse wake function for a circular aperture.

$$
\operatorname{Re} Z_{\|}{ }^{1}(\omega)=\frac{\kappa^{2}}{2 \pi \varepsilon_{0} c} \int_{a}^{b} K_{1}(\kappa r)^{2} \frac{1}{r} d r
$$

The dipole longitudinal coupling impedance is generally negligibly small compared to the monopole part. From it we can conveniently proceed to study the transverse wake. According to the Panofsky-Wenzel theorem [30], the transverse dipole impedance can be obtained,

$$
\operatorname{Re} Z_{\perp}{ }^{1}(\omega)=\frac{c}{\omega} \operatorname{Re} Z_{\|}{ }^{1}(\omega)=\frac{\kappa^{2}}{2 \pi \varepsilon_{0} \omega} \int_{a}^{b} K_{1}(\kappa r)^{2} \frac{1}{r} d r .
$$

The transverse wake is similarly calculated as

$$
W_{\perp}(s)=\frac{2}{\pi} \int_{0}^{\infty} d \omega \operatorname{Re} Z_{\perp}{ }^{1}(\omega) \sin \frac{\omega s}{c} .
$$

The transverse wake is calculated and shown in Fig. 10 for a circular DR target with $b / a=10$.

Here the convention is that $W_{\perp}(s)$ is positive when the kick is in the direction of the offset of the driving particle and the transverse wake has the dimension $\mathrm{V} / \mathrm{C} / \mathrm{m}$.

\section{PERTURBATIONS TO THE ELECTRON BEAM AND THE RESULTED PERFORMANCE DEGRADATION}

Every diagnostic method inevitably would cause perturbations to the beam and result in some degradation to the beam quality. Real-time monitoring beam parameters require that the degradation caused by the diagnosis does not lead to a significant reduction in performance of the facilities. Three typical applications with DR will be studied in this section: bunch length measurement for moderate energy beam as required by inverse Compton scattering based x-ray source; bunch length measurement after the first bunch compressor in an XFEL; and transverse profile 
measurement for high-energy beam. We assume the beam charge to be $1 \mathrm{nC}$ and normalized emittance to be $1 \mathrm{~mm}$. mrad for all three cases. For the first two cases, their bunch lengths are $1 \mathrm{ps}$ and energy is 50 and $250 \mathrm{MeV}$, respectively. For the third case, we assume the beam energy to be $5 \mathrm{GeV}$ and bunch length to be $0.2 \mathrm{ps}$. A circular disk with inner radius $2 \mathrm{~mm}$ and outer radius $20 \mathrm{~mm}$ will be used as the DR target for the first two cases and for the high-energy case, we will use a target with inner radius $1 \mathrm{~mm}$ and outer radius $10 \mathrm{~mm}$ in the calculation.

\section{A. Energy spread growth}

The average energy loss can be calculated as

$$
\Delta E_{\mathrm{av}}=\int_{-\infty}^{\infty} f(s) \Delta E(s) d s,
$$

and the rms energy spread growth is found to be

$$
\Delta E_{\mathrm{rms}}=\left[\int_{-\infty}^{\infty} f(s)\left[\Delta E(s)-\Delta E_{\mathrm{av}}\right]^{2} d s\right]^{1 / 2} .
$$

Before proceeding to calculate the specific values of the energy spread growth, it is worth discussing the features of the longitudinal bunch wake for the DR target. Let us consider a DR target with inner radius $2 \mathrm{~mm}$ and outer radius $20 \mathrm{~mm}$, the beam is assumed to be Gaussian with rms bunch length of $1 \mathrm{ps}$. The longitudinal bunch wake for various beam energy is found from Eq. (21) and shown in Fig. 11.

As we can see, when beam energy grows, the bunch wake tends to be resistive. It is worth pointing out that the resistive wake is generally related to very long bunch [31]. As can be seen in Fig. 7, the DR wake tends to be singular when the beam becomes ultrarelativistic. Therefore, even though the beam is quite short, when compared to the longitudinal wake, the beam becomes very long and the

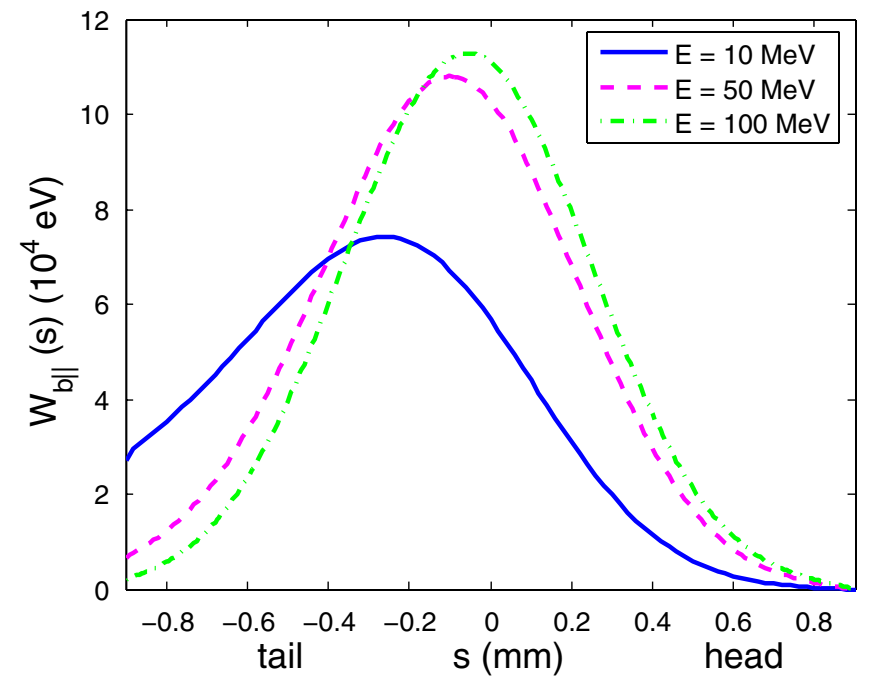

FIG. 11. (Color) Longitudinal bunch wake for electron beams with various energy. bunch wake becomes resistive. Also from Fig. 11 we could see that only when the beam tends to ultrarelativistic can their wake be independent of energy. As for the moderately relativistic beam, it is necessary to take into account the specific beam energy in the calculation of the wake.

The energy loss is only important for the low and moderate energy beam. The average energy loss and rms energy spread growth for the $50 \mathrm{MeV}$ case is found to be 60 and $23 \mathrm{keV}$. For the $250 \mathrm{MeV}$ case, the value is 81 and $31 \mathrm{keV}$, respectively.

For the inverse Compton scattering based $\mathrm{x}$-ray source, the energy spread growth will result in spectral broadening of the generated x-ray pulse. Since the x-ray energy scales in proportion to the square of the beam energy, we approximately have

$$
\frac{\Delta \omega}{\omega} \approx 2 \frac{\Delta E}{E}
$$

For the $50 \mathrm{MeV}$ case, the relative projected energy spread growth is found to be about $0.05 \%$ and the resulted spectral broadening is only $0.1 \%$, which is much smaller than that due to the laser bandwidth. So the use of DR to real-time monitor bunch length for the inverse Compton scattering facility is practicable. As for the $250 \mathrm{MeV}$ case, since only the slice energy spread is important for the lasing of an XFEL, the energy spread growth is still permissible.

\section{B. Emittance growth}

The transverse wake would add a position-dependent kick to the beam and results in emittance growth. The transverse bunch wake is calculated similar to Eq. (21),

$$
W_{b \perp}(s)=N e^{2} \int_{s}^{\infty} d s^{\prime} f\left(s^{\prime}\right) W_{\perp}\left(s^{\prime}-s\right) .
$$

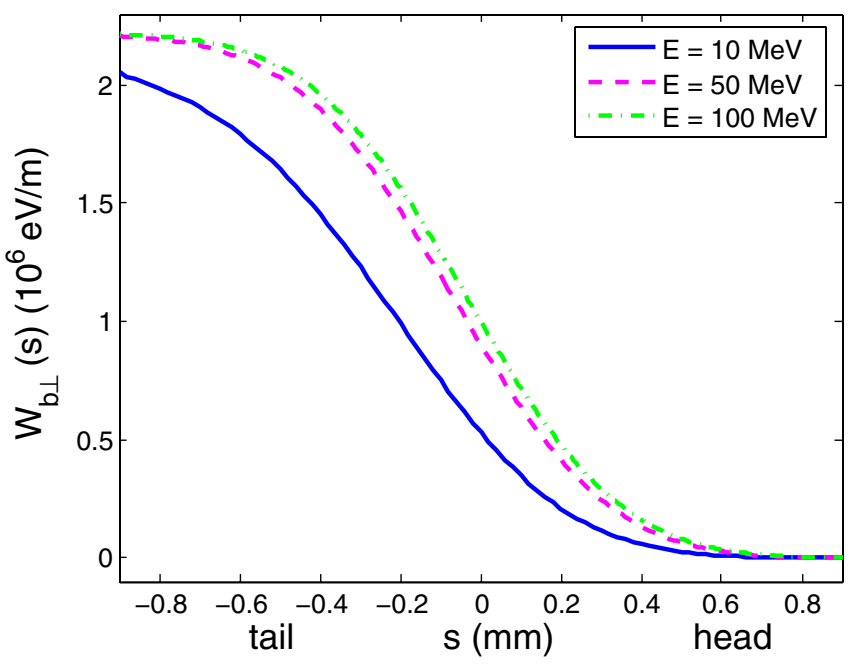

FIG. 12. (Color) Transverse bunch wake for electron beams with various energy. 
For the same parameters as in Fig. 11, the transverse bunch wake is calculated and shown in Fig. 12.

For the parameters used in Fig. 12, we found that the rms value of the bunch wake is about $624 \mathrm{keV} / \mathrm{m}$, which corresponds to a normalized emittance growth of about $0.07 \mathrm{~mm} \cdot \mathrm{mrad}$ if the beam's transverse size is taken to be $0.25 \mathrm{~mm}$ for the first two cases. As for the $5 \mathrm{GeV}$ case, we assume the transverse beam size to be $0.1 \mathrm{~mm}$, and the emittance growth is about $0.05 \mathrm{~mm} \cdot \mathrm{mrad}$. In all the cases of interest, the emittance growth due to use of the DR radiator is permissible.

\section{Trajectory deflection}

In real beam diagnostic setup, the DR target is tilted by 45 degrees with respect to the beam trajectory. In addition to the rms divergence increase due to the transverse wakefield from FDR, the BDR may add an average kick to the beam's trajectory as a result of momentum conservation. It is worth pointing out that this effect is not predicted by Maxwell's equations. It is a radiation recoil effect and cannot be handled with classically self-consistent theory and code.

The average deflection may be calculated as [6]

$$
r^{\prime}=\frac{\Delta p_{\perp}}{p}=\frac{1}{E} \iint \frac{d^{2} W \cos \theta}{d \omega d \Omega}[1+(N-1) F(\omega)] d \omega d \Omega,
$$

where $E$ is beam energy. The factor $\cos \theta$ in Eq. (30) takes into account the fact that the BDR propagates within a cone and only the perpendicular momentum affects the deflection while the parallel momentum cancels each other due to symmetry.

For the XFEL, the kick shifts the beam's trajectory and destroys the overlapping between the drive electron and amplified x-ray, which further reduces the FEL gain. This is similar to the effect of the misaligned quadruple from which the kick makes a nonstraight trajectory. The kick should be controlled to be less than the critical angle $\theta_{c}=$ $\sqrt{\lambda / L_{g}}$ in order not to cause serious performance degradation [32,33], where $\lambda$ is the amplified radiation wavelength and $L_{g}$ is the gain length. As for the ILC whose beam size is in the order of nm, any trajectory variation could destroy the collision and significantly reduce the luminosity.

Take the LCLS for example [34]; it can operate in either $4.54 \mathrm{GeV}$ with the aim of producing a $1.5 \mathrm{~nm}$ soft x-ray or $14.35 \mathrm{GeV}$ for a $0.15 \mathrm{~nm} x$-ray. The gain length for the $4.54 \mathrm{GeV}$ case is $1.3 \mathrm{~m}$ and that for $14.35 \mathrm{GeV}$ is $4.7 \mathrm{~m}$. The corresponding critical angle is found to be approximately 34 and $6 \mu \mathrm{rad}$ respectively. Considering a DR radiator with inner radius $1 \mathrm{~mm}$ and outer radius $10 \mathrm{~mm}$, beam charge of $1 \mathrm{nC}$, and rms bunch length of $70 \mathrm{fs}$; the kick is found to be about $119 \mu \mathrm{rad}$ for the $4.54 \mathrm{GeV}$ case and
$38 \mu \mathrm{rad}$ for the $14.35 \mathrm{GeV}$. The kick is much larger than the critical angle and is capable of completely destroying the FEL lasing. So we suggest a corrector put immediately downstream of the DR target to correct the kick. In this case the trajectory could be maintained straight and the DR could be used to perform real-time beam diagnosis.

As for the ILC, we use the typical parameters that beam energy is assumed to be $500 \mathrm{GeV}$, beam charge of $3.2 \mathrm{nC}$, and rms bunch length of 1 ps [35]. For the same DR radiator, even though due to the high energy and relatively long bunch length, the kick is found to be as small as $0.18 \mu \mathrm{rad}$, it still needs prompt correction considering the extremely small transverse size of the beam.

\section{SUMMARY AND CONCLUSIONS}

As compared to TR or other intercepting methods, the DR has the advantage that it avoids direct interaction with the beam and thus there is no limit on the beam intensity. While effectively avoiding heat deposition, Coulombic scattering, and bremsstrahlung as that happens in intercepting methods, one of the potential problems that may restrict its applications as a real-time monitoring method, if left unaddressed, is the wakefield generated by the DR radiator. In this paper, the DR and wakefield are studied near to the target with diffraction model and MAGIC simulation, respectively. It is found that the difference only exists in the subjective terminology. In typical applications the DR target is tilted by 45 degrees with respect to the beam's trajectory and the DR field is extracted out from the acceleration tube. Thus, the long range wakefield generated by the target should not affect the dynamics of the subsequent bunch. The short range wakefield would result in emittance growth and energy spread growth which is estimated and found to be permissible. However, the most serious problem is that the BDR propagates in the direction perpendicular to the trajectory and may add an average transverse kick to the beam as a requirement of momentum conservation. In order to use DR to real-time monitor beam parameters during accelerator operation, we suggest a corrector magnet be put immediately downstream of the DR radiator to compensate the kick and maintain a straight trajectory.

\section{ACKNOWLEDGMENTS}

We thank R. A. Bosch for helpful discussions and comments. One of the authors (D.X.) would like to thank G. Stupakov for sharing his note for calculating impedance by integration of the radiated energy in whole space. This work is supported by Chinese National Foundation of Natural Sciences under Contract No. 10475047.

[1] M. Castellano, Nucl. Instrum. Methods Phys. Res., Sect. A 394, 275 (1997). 
[2] A.H. Lumpkin, N.S. Sereno, and D. W. Rule, Nucl. Instrum. Methods Phys. Res., Sect. A 475, 470 (2001).

[3] M. Castellano, V. A. Verzilov, L. Catani, A. Cianchi, G. Orlandi, and M. Geitz, Phys. Rev. E 63, 056501 (2001).

[4] R. B. Fiorito and D. W. Rule, Nucl. Instrum. Methods Phys. Res., Sect. B 173, 67 (2001).

[5] Pavel Karataev, Sakae Araki, Ryosuke Hamatsu, Hitoshi Hayano, Toshiya Muto, Gennady Naumenko, Alexander Potylitsyn, Nobuhiro Terunuma, and Junji Urakawa, Phys. Rev. Lett. 93, 244802 (2004).

[6] Dao Xiang and Wen-Hui Huang, Phys. Rev. ST Accel. Beams 10, 012801 (2007).

[7] A. H. Lumpkin, W. J. Berg, N. S. Sereno, D. W. Rule, and C. Y. Yao, Phys. Rev. ST Accel. Beams 10, 022802 (2007).

[8] Dao Xiang, Wen-Hui Huang, and Yu-Zheng Lin, Phys. Rev. ST Accel. Beams 10, 062801 (2007).

[9] O. Grimm, Proceedings of PAC07, Albuquerque, 2007, p. 2653.

[10] R. Carr and H. Wiedemann, in Handbook of Accelerator Physics and Engineering, edited by A. W. Chao and M. Tigner (World Scientific, Singapore, 2006), 3rd ed., p. 217.

[11] Dao Xiang and Wen-Hui Huang, Nucl. Instrum. Methods Phys. Res., Sect. A 553, 381 (2005).

[12] Karl L.F. Bane and Gennady Stupakov, Phys. Rev. ST Accel. Beams 7, 064401 (2004).

[13] Bruce Goplen, Larry Ludeking, David Smith, and Gary Warren, Comput. Phys. Commun. 87, 54 (1995).

[14] Yu. N. Dnestrovskii and D. P. Kostomarov, Dokl. Akad. Nauk 124, 792 (1959).

[15] M.L. Ter-Mikaelian, High-Energy Electromagnetic Processes in Condensed Media (Wiley/Interscience, New York, 1972).

[16] R. A. Bosch, Phys. Rev. ST Accel. Beams 5, 020701 (2002).

[17] Dao Xiang and Wen-Hui Huang, Nucl. Instrum. Methods Phys. Res., Sect. B 248, 163 (2006).
[18] M. Born and E. Wolf, Principles of Optics (Cambridge University Press, Cambridge, England, 1999), 7th ed.

[19] H. A. Bethe, Phys. Rev. 66, 163 (1944).

[20] G. V. Stupakov, Wake and Impedance, Lecture presented at JAS 2000 Accelerator School On Frontiers Of Accelerator Technology, Moscow, Russia, 2000.

[21] S. A. Heifets and S. A. Kheifets, Rev. Mod. Phys. 63, 631 (1991).

[22] G. V. Stupakov (unpublished).

[23] I. S. Gradsbteyn and I. M. Ryzbik, Table of Integrals, Series and Products (Academic Press, New York, 2000), 6th ed.

[24] Mathematica, Wolfram Research, Inc.

[25] D. Davino, G. Miano, G. Panariello, and L. Verolino, Phys. Rev. ST Accel. Beams 2, 044401 (1999).

[26] G. V. Stupakov, Radiation of an Ultrarelativistic Particle Passing through a Round Hole in a Perfectly Conducting Screen, http://www.slac.stanford.edu/ stupakov/.

[27] G. Stupakov, K. L. F. Bane, and I. Zagorodnov, Phys. Rev. ST Accel. Beams 10, 054401 (2007).

[28] R. A. Bosch, Phys. Rev. ST Accel. Beams 10, 050701 (2007).

[29] A. W. Chao, Physics of Collective Beam Instabilities in High Energy Accelerators (Wiley, New York, 1993).

[30] W. K. H. Panofsky and W. A. Wenzel, Rev. Sci. Instrum. 27, 967 (1956).

[31] P. Morton, Report No. SLAC-PUB-6052 (1993).

[32] T. Tanaka, H. Kitamura, and T. Shintake, Nucl. Instrum. Methods Phys. Res., Sect. A 528, 172 (2004).

[33] Zhirong Huang and Kwang-Je Kim, Phys. Rev. ST Accel. Beams 10, 034801 (2007).

[34] LCLS Conceptual Design Report, Report No. SLAC-R593, 2002.

[35] International Linear Collider Reference Design Report, 9th ACFA ILC Physics and Detector Workshop \& ILC GDE Meeting, Beijing, 2007. 